\title{
Optimal penalty method in distribution service restoration using genetic algorithm
}

\begin{abstract}
As an efficient scheme for service restoration in distribution systems has a vital role in improving reliability of the system and also satisfactory of customers, significant efforts assigned in solving the problem. The major challenge is to reduce computation burden while covering all the possible answers in reasonable time and effort. Furthermore, restoration is a multi-objective, multi-constraint optimization problem. The considered restoration objective functions in this study include the minimization of outage area, minimizing of power loss and minimizing of number of switching whilst considering the technical constraints. This study presents a new approach of supply restoration service using the Genetic Algorithm (GA). A new hybrid Genetic Algorithm is proposed for reducing the search space. The proposed technique is implemented to improve the penalty strategy to enhance the performance of algorithm and reduce the convergence iteration. The effectiveness of the proposed method is demonstrated by testing on a 33-bus test system. Comparisons show the improvements in reducing of number of iteration after restoration. Findings through comparisons are shown that the proposed method will be able to do full restoration and energize all loads.
\end{abstract}

Keyword: Service restoration; Distribution systems; Genetic algorithm; Penalty method 